Original Article

\title{
Long-Term Outcome of Active Infective Endocarditis with Renal Insufficiency in Cardiac Surgery
}

\author{
Kiyoshi Tamura, MD, ${ }^{1,2}$ Hirokuni Arai, MD, ${ }^{2}$ and Tomoya Yoshizaki, $\mathrm{MD}^{1}$
}

\begin{abstract}
Background: The relation between infective endocarditis (IE) and renal insufficiency is uncertain. The aim of this study was to investigate active IE with renal insufficiency in cardiac surgery.

Patients and Methods: A retrospective record review was conducted of all cases with IE from January 1998 to July 2009. We identified 38 patients who had undergone surgical intervention (25 males and 13 females, mean age $57.3 \pm 15.2$ years, range $23-83$ years) of IE as defined by the modified Duke criteria. Indications for surgical intervention included new, severe valvular regurgitation with heart failure, intracardiac abscesses, and recurrent embolic events. All patients were divided two groups; one group comprised patients without renal insufficiency (group $N, n=28$ ), the other, those with renal insufficiency (group $R, n=10$ ). Results: Mean age of patients in group $R$ was larger than that in of group $N(66.3 \pm 10.6$ vs. $54.1 \pm 15.4$ years, $p=0.0268$ ), and mean hemoglobin in group $R$ than in group $N(8.4 \pm 0.9$ vs. $10.3 \pm 2.5 \mathrm{~g} / \mathrm{dl}, \mathrm{p}=\mathbf{0 . 0 2 1 5}$ ). In the early outcome, hospital death was greater in group $\mathrm{R}$ than in group $\mathrm{N}(20.0 \%$ vs. $0.0 \%, p=0.0143)$. The 8 -year survival was significantly worse in group $R$ than in group $N(50.0 \%$ vs. $96.4 \%$, log rank test: $p=0.0042)$. Moreover, the 8-year actuarial freedom from cardiac events was significantly worse in group $R$ than in group $\mathbf{N}(0.0 \%$ vs. $60.3 \%$, log rank test: $p=\mathbf{0 . 0 0 0 3}$ ), too. Renal insufficiency predicted an increase in long-term mortality $(\mathrm{OR} 12.104,95 \% \mathrm{CI} 1.349-108.641, \mathrm{p}=0.0259)$ and morbidity $(\mathrm{OR} 10.540,95 \% \mathrm{CI}$ 2.173-51.129, $p=0.0035)$.

Conclusions: In IE, renal insufficiency may allow for risk stratification of patients undergoing surgical intervention.
\end{abstract}

Keywords: infective endocarditis, valvular disease, microorganism, renal insufficiency

\footnotetext{
${ }^{1}$ Department of Cardiovascular Surgery, Musashino Red Cross Hospital, Tokyo, Japan

${ }^{2}$ Department of Cardiovascular Surgery, Tokyo Medical and Dental University, Tokyo, Japan
}

Received: July 26, 2011; Accepted: November 7, 2011 Corresponding author: Kiyoshi Tamura, MD. Department of Cardiovascular Surgery, Tokyo Medical and Dental University, 1-5-45 Yushima, Bunkyo-ku, Tokyo 113-8519, Japan

Email: tamura.cvsg@tmd.ac.jp

(C)2012 The Editorial Committee of Annals of Thoracic and Cardiovascular Surgery. All rights reserved.

\section{Introduction}

Acute infective endocarditic (IE) is a clinical challenge that is often associated with high patient morbidity and mortality. ${ }^{1)}$ Although IE is generally treated using antibiotics, surgical intervention is required in $20 \%$ of cases. ${ }^{2)}$ Despite outcome improvements due to advances in antibiotic therapy, diagnosis, and general treatment, IE results in high morbidity and mortality.

Indications for surgery in IE have continued to evolve, but frequently include intractable heart failure, abscess formation, recurrent embolic events, organism involved, and presence of prosthetic material. ${ }^{4-9)}$ While mortality 
has improved from $30 \%$ to $15 \%$ over the past two decades, it remains substantial. ${ }^{1}$

With increasing numbers of patients with indications for IE surgery, the identification of preoperative variables that may identify the highest risk patients (example as renal insufficiency) in this surgical cohort would be clinically useful. Although prior studies have evaluated clinical, microbiological, and echocardiographic predictors of patients with IE, ${ }^{10-14)}$ little is known about long-term outcomes of patients with renal insufficiency.

We thus sought to evaluate the impact of renal insufficiency on morbidity and mortality in patients with surgical intervention of IE.

\section{Patients and Methods}

We retrospectively reviewed all cases of IE diagnosed at Musashino Red Cross Hospital, from January 1998 to July 2009. Only patients with definite IE according to the modified Duke criteria were included. ${ }^{4)}$ During the study period, all patients with bacteremia were evaluated by infectious disease staff members. The following data were analyzed at the time of diagnosis: demographic features, predisposing heart diseases and past personal history, clinical features, the affected valves, bacteriologic diagnosis, echocardiographic studies, radiologic images, results of routine laboratory tests (including hematological value, liver and renal function, and inflammatory parameters), treatment and outcome. The study protocol was approved by the ethics broad of our hospital. All patients were informed about the procedure, and they provided written confirmation for the operation.

The indications for surgery in IE included intractable heart failure, abscess formation, recurrent embolic events, and organism involved. We provided antibiotics and/or surgical intervention followed the guideline of the Japanese Circulation Society.

Cardiac event was defined as a cardiac death, re-cardiac operation, heart failure, and cerebral vascular accidents. Renal insufficiency was defined as patients with hemodialysis or creatinine $>1.5 \mathrm{mg} / \mathrm{dl}$.

All patients were divided two groups; one group comprised patients without renal insufficiency (group N), the other, those with renal insufficiency (group R). The following data of the two groups were compared to evaluate predictors of morbidity and mortality in patients with IE.

Indications for surgical intervention included new, severe valvular regurgitation with heart failure, intracardiac abscesses, and recurrent embolic events. However, we ex-
Table 1 Surgical intervention for infective endocarditis

\begin{tabular}{lc}
\hline Operation & Patient number \\
\hline AVR & 9 \\
MVR & 9 \\
AVR + MVR & 6 \\
TVR & 4 \\
MVP & 2 \\
AVR + TVR & 1 \\
AVR + PDA closure & 1 \\
re-AVR & 1 \\
re-MVR & 1 \\
MVR + TAP & 1 \\
MVR + TVR & 1 \\
MVR + VSD closure & 1 \\
VSD closure & 1 \\
\hline
\end{tabular}

AVR: aortic valve replacement; MVR: mitral valve replacement; TVR: tricuspid valve replacement; MVP: mitral valve plasty; PDA: patent ductus arteriosus; TAP: tricuspid valve annuloplasty; VSD: ventricular septal defect

cluded patients with consciousness dysfunction from surgical indication.

Continuous data are expressed as mean \pm SD with ranges when appropriate. Comparisons between two categories variables were made using the Student's $t$ test for continuous variables. Otherwise, non-parametric MannWhitney $U$ test was used. Parametric data were examined with contingency tables, with Fisher's exact test, as appropriate. The follow-up survival was assessed using the Kaplan-Meier survival curve and log rank test. Differences were considered significant at $p<0.05$. Statistical analysis was performed with StatView for Windows version 6.0 (SAS Institute Inc, Cary, NC).

\section{Results}

During the study period, there were 71 patients with diagnosis of IE as defined by the modified Duke criteria. Of all, the 38 patients ( 25 males and 13 females, mean age $57.3 \pm 15.2$ years, range $23-83$ years) were performed surgical intervention during their initial hospitalization, and the operations were shown in Table 1. Mean length of operation from diagnosis was $13.4 \pm 12.7$ days (range 1-52 days).

All patients were divided two groups; one comprised patients without renal insufficiency ( $\mathrm{N}$ group, $\mathrm{n}=28$ ); the other, those with renal insufficiency ( $\mathrm{R}$ group, $\mathrm{n}=10$ ).

\section{Demographic characteristics}

The characteristics at hospital arrival were shown in 
Table 2 Demographic characteristics of 38 patients with infective endocarditis

\begin{tabular}{|c|c|c|c|}
\hline & $\begin{array}{l}\text { N group } \\
n=28\end{array}$ & $\begin{array}{l}\text { R group } \\
\mathrm{n}=10\end{array}$ & $\mathrm{p}$ value \\
\hline \multicolumn{4}{|l|}{ Demographic variables } \\
\hline Female & $11(39.3 \%)$ & $2(20.0 \%)$ & 0.2822 \\
\hline Age (years) & $54.1 \pm 15.4$ & $66.3 \pm 10.6$ & 0.0268 \\
\hline Diabetes mellitus & $2(7.1 \%)$ & $1(10.0 \%)$ & 0.7809 \\
\hline Immunosuppressores & $0(5.0 \%)$ & $1(10.0 \%)$ & 0.0946 \\
\hline Embolic event & $16(57.1 \%)$ & $5(50.0 \%)$ & 0.7059 \\
\hline \multicolumn{4}{|l|}{ Laboratory data at hospital arrival } \\
\hline Leukocyte (/mm3) & $11492 \pm 5178$ & $11790 \pm 5015$ & 0.8766 \\
\hline Hemoglobin (g/dl) & $10.3 \pm 2.5$ & $8.4 \pm 0.9$ & 0.0215 \\
\hline $\mathrm{CRP}(\mathrm{mg} / \mathrm{dl})$ & $10.2 \pm 8.9$ & $10.8 \pm 8.2$ & 0.8504 \\
\hline Cre (mg/dl) & $0.80 \pm 0.26$ & $2.78 \pm 1.42$ & $<0.0001$ \\
\hline Valve involvement & $13(46.4 \%)$ & $5(50.0 \%)$ & 0.8511 \\
\hline Aortic & $16(57.1 \%)$ & $6(60.0 \%)$ & 0.8793 \\
\hline Mitral & $4(14.3 \%)$ & $3(30.0 \%)$ & 0.2836 \\
\hline Tricuspid & $3(10.7 \%)$ & $0(0.0 \%)$ & 0.2934 \\
\hline $\begin{array}{l}\text { Congenital cardiac disease } \\
\text { Prosthetic Valve }\end{array}$ & $2(7.1 \%)$ & $0(0.0 \%)$ & 0.3989 \\
\hline $\begin{array}{l}\text { Echocardiogram } \\
\text { LVEF }\end{array}$ & $71.7 \pm 10.3$ & $75.1 \pm 13.4$ & 0.6151 \\
\hline Outcome & $0(0.0 \%)$ & $2(20.0 \%)$ & 0.0143 \\
\hline Hospital death & $55.4 \pm 31.4$ & $55.7 \pm 23.2$ & 0.9776 \\
\hline \multicolumn{4}{|l|}{ Hospital stay (days) } \\
\hline \multicolumn{4}{|l|}{ Migroorganism } \\
\hline Gram-positive & $11(39.3 \%)$ & $6(60.0 \%)$ & 0.2703 \\
\hline Staphylococcus & $9(32.1 \%)$ & $1(10.0 \%)$ & 0.1815 \\
\hline Streptococcus & $0(0.0 \%)$ & $0(0.0 \%)$ & \\
\hline Gram-negative & $3(10.7 \%)$ & $2(20.0 \%)$ & 0.3278 \\
\hline $\begin{array}{l}\text { Other microorganisms } \\
\text { Culture negative }\end{array}$ & $5(17.9 \%)$ & $1(10.0 \%)$ & 0.571 \\
\hline
\end{tabular}

CRP: C-reactive protein; LVEF: left ventricular ejection fraction

Table 2. Of all patients, the majority (94.7\%) were diagnosed with native valve endocarditis. Patients in group $\mathrm{R}$ were significantly older than those in group $\mathrm{N}(66.3 \pm$ 10.6: $54.1 \pm 15.4$ years, $\mathrm{p}=0.0268$ ). Diabetes mellitus and immunosupressores were not different in the both groups. Though patients with an embolic event were $55.3 \%(\mathrm{n}=$ 21) of all, there was no difference between both groups.

At hospital arrival, the mean hemoglobin value of group $\mathrm{R}$ was lower than that of group $\mathrm{N}(8.4 \mathrm{~g} / \mathrm{dL} \pm 0.9$ vs. $10.3 \mathrm{~g} / \mathrm{dL} \pm 2.5, \mathrm{p}=0.0215$ ), but the inflammatory examination (leukocyte, CRP) was not different in both groups.

There was no difference in valve involvement. Prosthetic valve infection was two patients (5.3\%). Then, left ventricular function was not different between two groups, too.

There were 2 hospital deaths (5.3\%) in all patients, and two patients were in group R. One was sepsis, and the other was multiple organ failure. Hospital death was more in group $\mathrm{R}$ than in group $\mathrm{N}(20.0 \%$ vs. $0.0 \%, \mathrm{p}=0.0143)$. The hospital stay of all patients was a mean of $55.5 \pm 29.1$ days.

Organisms isolated from 38 patients is shown in Table 3. The majority of patients ( 27 patients, $71.1 \%$ ) were isolated Gram-positive microorganism, and culture negative was in 6 patients (15.8\%). There was no negative microorganism isolated in this study.

\section{Long-term evaluation}

Follow-up was completed in 37 patients (97.3\%). The average follow-up period was $37.4 \pm 33.3$ months (range 2 to 139 months). With the exclusion of hospital deaths, two patients of all died, and both causes of death were cerebral bleeding at 11 months and 35 months after their hospital discharge.

Survival rates (including hospital death) by KaplanMeier curve are shown in Fig. 1. In group N, the 1, 5, 8 -year survivals were $96.4 \%, 96.4 \%$, and $96.4 \%$. In 
Table 3 Hazard ratio for total population, according to long-term outcome

\begin{tabular}{lccc}
\hline Characteristics for survival & Odds ratio & $95 \%$ confidence intervals & $\mathrm{p}$ value \\
\hline Over 70 year old & 2.954 & $0.267-32.627$ & 0.3768 \\
Female & 1.535 & $0.160-14.757$ & 0.7108 \\
Renal insufficiency & 12.104 & $1.349-108.641$ & 0.0259 \\
Aortic valve infection & 1.761 & $0.293-10.579$ & 0.536 \\
Mitral valve infection & 0.153 & $0.017-1.374$ & 0.0936 \\
Embolic event & 0.178 & $0.020-1.600$ & 0.1232 \\
Staphylococcus infection & 1.787 & $0.297-10.753$ & 0.5261 \\
\hline & & & \\
\hline Characteristics for cardiac event & Odds ratio & $95 \%$ confidence intervals & value \\
\hline Over 70 year old & 4.117 & $0.684-24.778$ & 0.1223 \\
Female & 2.102 & $0.441-10.021$ & 0.3513 \\
Renal insufficiency & 10.54 & $2.173-51.129$ & 0.0035 \\
Aortic valve infection & 1.14 & $0.328-3.958$ & 0.8367 \\
Mitral valve infection & 0.988 & $0.277-3.254$ & 0.9856 \\
Embolic event & 0.644 & $0.184-2.253$ & 0.4912 \\
Staphylococcus infection & 1.507 & $0.413-5.494$ & 0.5345 \\
\hline
\end{tabular}

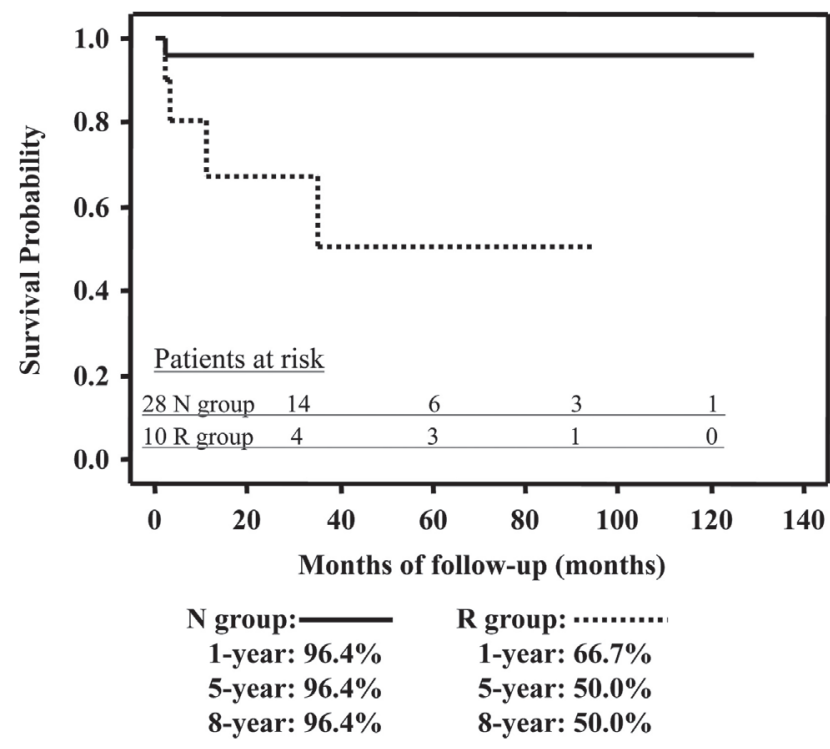

Log rank test: $p=0.0042$

Fig. 1 Actuarial survival rates estimated by Kaplan-Meier analysis.

contrast, the 1, 5, 8-year survivals were $66.7 \%, 50.0 \%$, and $50.0 \%$ in group R. Survival was significantly worse in group $\mathrm{R}$ than in group $\mathrm{N}(\log$ rank test, $\mathrm{p}=0.0042)$.

Then, the cardiac event-free rate by the Kaplan-Meier curve is shown in Fig. 2. In group N, the 1, 5, 8-year cardiac event-free rates were $96.4 \%, 80.4 \%$, and $60.3 \%$. On the other hand, the 1, 5, 8-year cardiac event-free rate were $56.0 \%, 42.0 \%$, and $0.0 \%$ in group $\mathrm{R}$. The actuarial freedom from cardiac events was significantly worse in

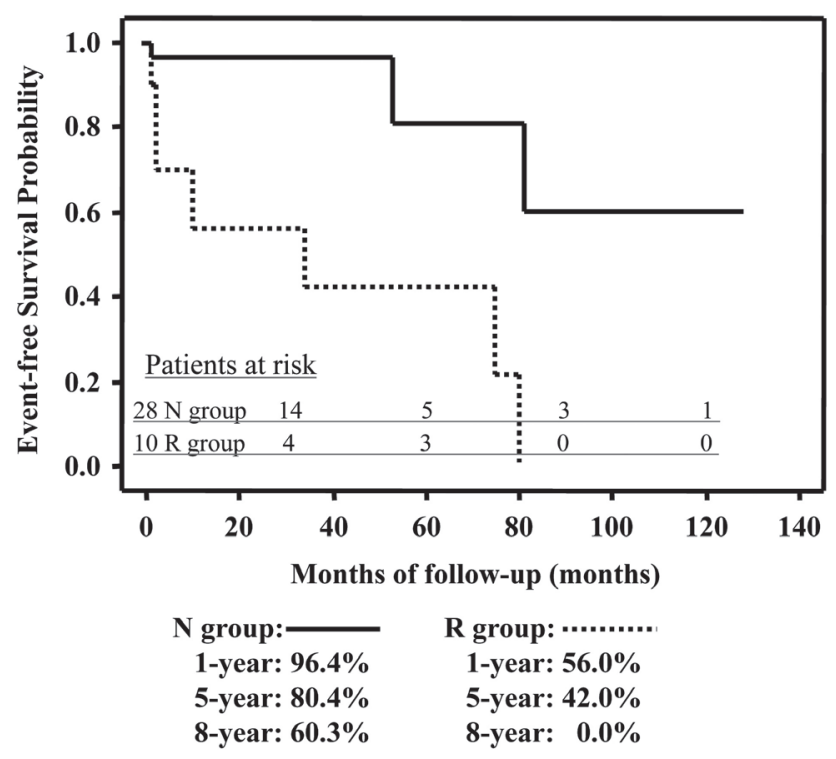

Log rank test: $\mathbf{p}=\mathbf{0 . 0 0 0 3}$

Fig. 2 Actuarial cardiac-related event-free survival rates estimated by Kaplan-Meier analysis.

group R than in group $\mathrm{N}(\log$ rank test: $\mathrm{p}=0.0003)$, too.

\section{Predictors of cardiac event}

We investigated whether perioperative factors were predictors of long-term survival by multivariable analysis. Table 3 shows the Hazard ratio of long-term survival. Only renal insufficiency (odds ratio 12.104, 95\% confidence interval 1.349-108.641; $\mathrm{p}=0.0259$ ) was indicated predictors of long-term survival in patients with surgical 
intervention of IE.

In the same way, we investigated predictors of cardiac event by multivariable analysis. Table 3 shows the hazard ratio for the cardiac event. Similarly, renal insufficiency was a unique predictor of the cardiac event (odds ratio 10.540, 95\% confidence interval 2.173-51.129; $\mathrm{p}=$ 0.0035).

For both survival and the cardiac event, renal insufficiency was significantly associated with the long-term evaluation.

\section{Discussion}

In the present study, we have shown that the survival rate and cardiac-event free rate were fully satisfied and that renal insufficiency was associated with high mortality and morbidity in patients with surgical intervention of IE.

Recent uncontrolled observational studies have suggested that early surgery is more beneficial for patients with IE compared with medical therapy alone..$^{11,15,16)}$ Thus, surgery is currently performed in at least $25 \%$ of patients with active $\mathrm{IE}^{12)}$ and in $50 \%$ of patients, respectively, in another study. ${ }^{17)}$ In our study, cardiac surgery was performed in $53.5 \%$ of all patients with IE. The hospital death rate was $5.3 \%$ in patients who had a surgical intervention during their initial hospitalization, though the hospital death rate was $24.2 \%$ in patients who did not have a surgical intervention (data not shown).

Hoen and colleagues ${ }^{18)}$ suggested that the decrease in the rate of in-hospital mortality observed in a 10-year interval may have been related to a higher rate of cardiac surgery. In a recent large prospective study including 267 patients with left side $\mathrm{IE}^{12)}$ as in other previous series $8,{ }^{19-21)}$ early cardiac surgery was not associated with lower in-hospital mortality. Decisions about surgery are often problematic owing to the lack of evidence from randomized controlled studies. In most cases, through consultation between the medical and the surgical teams is necessary to analyze the best strategy. Surgery in our study was performed according to conventional guidelines, predominantly in the presence of complications. However, Vikram and colleagues ${ }^{17)}$ found that, in patients with left-side complicated IE, surgery was an independent predictor of 6-month survival. Reduced mortality related to surgery was particularly significant among patients with congestive heart failure. In the current study, early surgery in Staphylococcus aureus IE was associated with reduced in-hospital mortality in a univariate model with a trend in multivariate analysis toward reduction of in-hospital mortality $(\mathrm{p}=0.1)$. Moreover, early surgery was independently related to a reduction in overall mortality.

Renal insufficiency in our study was found to be a prognostic factor associated with higher mortality and morbidity rates. A number of theories about the mechanisms for increased mortality and morbidity among renal failure patients have been proposed. Dialysis-dependent renal failure patients are an immune compromised population, and this may contribute to their increased risk of postoperative infection. For example, dialysis-dependent renal failure patients have a higher wound infection rate after a lower extremity bypass. ${ }^{22)}$ Whether increased infection rates are due to uremia, diabetes, steroid use secondary to autoimmune causes of renal failure, and other variables that are known to increase the risk of infection is unknown. Dialysis patients are also chronically anemic from the loss of erythropoietin production by the kidneys, ${ }^{23,24)}$ and anemia has been shown to be associated with increased risks of mortality among patients with coronary artery bypass grafting. ${ }^{25)}$ Platelet dysfunction and coagulation defects from uremia are cited as mechanisms for increased bleeding among patients on dialy$\mathrm{sis}^{26)}$ and renal failure may serve as a maker for generalized arteriosclerosis predisposing to thromboembolic events and stroke, ${ }^{27)}$ as well as to less adequate revascularization and development of postoperative low-output syndrome. ${ }^{28)}$ In this study, preoperative hemoglobin in patients with renal insufficiency was lower than in patients without renal insufficiency (Table 2), and we expected that anemia- induced renal insufficiency might be one of factors affected short- and long-term outcomes.

\section{Study limitations}

The results of this study should be interpreted in light of certain limitations. Firstly, ours is a retrospective study. Nevertheless, we believe that the prevalence of IE was below the true rate, due to the retrospective nature of this study. Secondly, the present study was a single-center experience, and, as a result, was limited by the relatively small number of patients. Despite these limitations, our study provides predictors of long-term outcomes in patients with IE.

\section{Conclusion}

This study shows that renal insufficiency may allow for long-term risk stratification of patients with surgical intervention of IE. 


\section{Disclosure Statement}

We report no conflicts of interest.

\section{References}

1) Mylonakis E, Calderwold SB. Infective endocarditis in adults. N Engl J Med 2001; 345: 1318-30.

2) Bayer AS, Schled WM. Endocarditis and intravascular infections. In: Mandell GL, editor. Principles of infectious disease. Fifth editor. Churchill Livingstone: Eninburgh, 2000; 857-902.

3) Moreillon P. Endocarditis and endarteritis. In: Infectious diseases. Armstrong D, Cohen J, editor. London: Harcourt Publishers; 1999; 2.50.1-2 50.9.

4) Li JS, Sexton DJ, Mick N, et al. Proposed modifications to the Duck criteria for the diagnosis of infective endocarditis. Clin Infect Dis 2000; 30: 633-8.

5) Daniel WG, Mugge A, Martin RP, et al. Improvement in the diagnosis of abscesses associated with endocarditis by transesophageal echocardiography. N Engl J Med 1991; 324: 795-800.

6) Yvorchuk KJ, Chan KL. Application of transthoracic and transesophageal echocardiography in the diagnosis and management of infective endocarditis. J Am Soc Echocardiogr 1994; 7: 294-308.

7) Bonow RO, Carabello B, de Leon AC Jr, et al. Guidelines for the management of patients with valvular disease: a report of the American College of Cardiology/ American Heart Association Task Force on Practice Guidelines (Committee on Management of Patients with Valvular Disease). Circulation 1998; 98: 1949-84.

8) Bayer AS, Bolger AF, Taubert KA, et al. Diagnosis and management of infective endocarditis and its complications. Circulation 1998; 98: 2936-48.

9) Douglas JL, Cobbs GG. Prosthetic valve endocarditis. In Kaye D, ed. Infective endocarditis. 2nd ed. New York: Raven Press; 1992; 375-396.

10) Cabell CH, Pond KK, Peterson GE, et al. The risk of stroke and death in patients with aortic and mitral valve endocarditis. Am Heart J 2001; 142: 75-80.

11) Hasbun R, Vikram HR, Barakat LA, et al. Complicated left-side native valve endocarditis in adults: risk classification for mortality. JAMA 2003; 289: 1933-40.

12) Chu VH, Cabell CH, Bejamin DK Jr, et al. Early predictors of in-hospital death in infective endocarditis. Circulation 2004; 109: 1745-9.

13) De Castro S, Mangi G, Beni S, et al. Role of transthoracic and transesophageal echocardiography in predicting embolic events in patients with active infective endocarditis involving native cardiac valves. Am J Cardiol 1977; 80: 1030-4.
14) Sanifilippo AJ, Picard MH, Newell JB, et al. Echocardiographic assessment of patients with infective endocarditis: prediction of risk for complication. J Am Coll Cardiol 1991; 18: 1191-9.

15) Netzer ROM, Altwegg SC, Zollinger E, et al. Infective endocarditis: determinants of long term outcome. Heart 2002; 88: 61-6.

16) Vlessis AA, Hovaguimian H, Jaggers J, et al. Infective endocarditis: ten-year review of medical and surgical therapy. Ann Thorac Surg 1996; 61: 1217-22.

17) Vikram HR, Buenconsejo J, Hasbun R, et al. Impact of valve surgery on 6-month mortality in adults with complicated, left-sided native valve endocarditis: a propensity analysis. JAMA 2003; 290: 3207-14.

18) Hoen B, Alla F, Selton-Suty C, et al. Changing profile of infective endocarditis. Results of a 1-year survey in France. JAMA 2002; 288: 75-81.

19) Acar J, Michel PL, Varenne O, et al. Surgical treatment of infective endocarditis. Eur Heart J 1995; 16 (Suppl B): 94-8.

20) Kirklin JK, Kirklin JW, Pacifico AD. Aortic valve endocarditis with aortic root abscess cavity: surgical treatment with aortic valve homograft. Ann Thorac Surg 1988; 45: 674-7.

21) Glazier JJ, Verwilghen J, Donaldson RM, et al. Treatment of complicated prosthetic aortic valve endocarditis with annular abscess formation by homograft aortic root replacement. J Am Coll Cardiol 1991; 17: 1177-82.

22) Johnson BL, Glickman MH, Bandyk DF, et al. Failure of foot salvage in patients with end-stage renal disease after surgical revascularization. J Vasc Surg 1995; 22: 280-5. discussion 285-6.

23) Pastan S, Bailey J. Dialysis theraphy. N Engl J Med 1998; 338: 1428-37.

24) Morbidity and mortality of renal dialysis: an NIH consensus conference statement. Ann Intern Med 1994; 121: $62-70$.

25)DeFoe GR, Ross CS, Olmstead EM, et al. Lowest hematocrit on bypass and adverse outcomes associated with coronary artery bypass grafting. Northern New England Cardiovascular Disease Study Group. Ann Thorac Surg 2001; 71: 769-76.

26)Jahangiri M, Wright J, Edmondson S, et al. Coronary artery bypass grafting surgery in dialysis patients. Heart 1997; 78: 343-5.

27) John R, Choudhri AF, Weinberg AD, et al. Multicenter review of preoperative risk factors for stroke after coronary artery bypass grafting. Ann Thorac Surg 2000; 69: 30-5. discussion 35-6.

28) Rao V, Weisel RD, Buth KJ, et al. Coronary artery bypass grafting in patients with non-dialysis-dependent renal insufficiency. Circulation 1997; 96 (9 Supple): II38-II-45. 